\title{
How many satellites have been discovered in the Solar System after Galileo?
}

\author{
Zhanna Pozhalova $^{1}$ and Dmitrij Lupishko ${ }^{2}$ \\ ${ }^{1}$ Research Institute "Nikolaev Astronomical Observatory", \\ Observatornaya 1, Nikolaev, 54030, Ukraine \\ email: zhanna@mao.nikolaev.ua \\ ${ }^{2}$ Institute of Astronomy of Kharkiv V. N. Karazin National University, \\ Sumskaya 35, Kharkiv, 61001, Ukraine \\ email: lupishko@astron.kharkov.ua
}

\begin{abstract}
By the beginning of 2010 the total number of natural satellites and multiple systems in the Solar System was equal to 350, including: 168 satellites of large planets, 119 multiple asteroids (including main-belt and near-Earth asteroids, Mars-crossers and Jupiter Trojan asteroids) and 63 multiple transneptunian and Kuiper-belt objects. Meanwhile, we cannot count precisely how many moons in total have been discovered to date due to the deficiency of accepted definitions.
\end{abstract}

Keywords. Planets and satellites, binary asteroids, discoveries.

\section{Discoveries}

The Moon as a natural satellite of the Earth was "discovered" by Nicolaus Copernicus (1473-1543) in his heliocentric system and it was the only known satellite in the Solar System before Galileo Galilei (1564-1642). At the end of 1609 Galileo had made the new adjustment to his instrument and turned his attention to Jupiter. Early in 1610 he discovered four satellites orbiting Jupiter, that are the satellites of other planet than the Earth. They are well known as Galilean moons (or satellites) Io, Europa, Ganymede and Callisto. His book, Sidereus Nuncius, in which his discovery was described, came off the press in Venice in the middle of March 1610 and made Galileo famous.

It was not until 45 years later (13 years after Galileo's death) that the next satellite in the Solar System, namely the biggest Saturn's moon Titan, was discovered by Christian Huygens (1629-1695). He first "published" his discovery as an anagram, sent out on June 13, 1655; later he published in pamphlet form as De Saturni luna Observatio Nova and in full in Systema Saturnium (July 1659). After that the discoveries of satellites happened more frequently and by the end of the $17^{\text {th }}$ century the total number of discovered moons was ten. Giovanni Domenico Cassini (1625-1712) was the first to discover the four of Saturn's moons (Iapetus in 1671; Rhea in 1672; Tethys and Dione in 1684).

From 1684 till 1787 (during more than one century!) no satellites were discovered and the next $18^{\text {th }}$ century added only four satellites (two of Uranus and two of Saturn). They were discovered by William Herschel (1738-1822), the most famous astronomer of the $18^{\text {th }}$ century, who constructed more than four hundred telescopes and using his own telescopes, discovered Titania and Oberon in 1787 as well as Enceladus and Mimas in 1789. Herschel also discovered the $7^{t h}$ planet Uranus in 1781. It should be stressed that during that century there were no more discoveries of either planets or satellites. Thus, we can conclude that while Galileo Galilei was the greatest discoverer of the $17^{\text {th }}$ century, W. Herschel was the greatest discoverer of the $18^{\text {th }}$ century. 
Table 1. Timeline of planet satellite's discoveries.

\begin{tabular}{llllllll}
\hline Planet & $\begin{array}{l}\text { Number } \\
\text { of moons }\end{array}$ & $\begin{array}{l}\mathbf{1 7}^{t h} \\
\text { century }\end{array}$ & $\begin{array}{l}\mathbf{1 8}^{\text {th }} \text { century } \\
\text { century }\end{array}$ & $\begin{array}{l}\mathbf{1 9}^{\text {th }} \\
\text { century }\end{array}$ & $\begin{array}{l}\mathbf{2 0}^{\text {th }} \\
\text { century }\end{array}$ & $\begin{array}{l}\mathbf{2 1}^{t h} \\
\text { century }\end{array}$ & $\begin{array}{l}\text { By space } \\
\text { mission }\end{array}$ \\
\hline Earth & 1 & & & & & & \\
Mars & 2 & & 2 & & & \\
Jupiter & 63 & 4 & & 1 & 13 & 45 & 3 \\
Saturn & 62 & 5 & 2 & 2 & 21 & 32 & 12 \\
Uranus & 27 & & 2 & 2 & 17 & 6 & 11 \\
Neptune & 13 & & & 1 & 7 & 5 & 6 \\
Total & 168 & 9 & 4 & 8 & 58 & 88 & 32 \\
\hline
\end{tabular}

Table 2. Sizes and masses of the biggest satellites.

\begin{tabular}{llllllll}
\hline & Ganymede & Titan & Callisto & Io & Moon & Europa & Triton \\
\hline $\mathrm{R}(\mathrm{km})$ & 2631 & 2575 & 2403 & 1815 & 1737,4 & 1569 & 1350 \\
Mass $(\mathrm{kg})$ & $1.48 \cdot 10^{23}$ & $1.35 \cdot 10^{23}$ & $1.08 \cdot 10^{23}$ & $8.94 \cdot 10^{22}$ & $7.35 \cdot 10^{22}$ & $4.8 \cdot 10^{22}$ & $2.14 \cdot 10^{22}$ \\
\hline
\end{tabular}

In the $19^{\text {th }}$ century the $8^{\text {th }}$ planet Neptune and its moon Triton, two Uranus' and two Saturn's moons, one of Jupiter's and both of Mars' moons (Phobos and Deimos) were discovered. The available theoretical considerations guided Asaph Hall (1829-1907) to conclusion that any Martian satellite must revolve very close to the planet. He undertook systematic search for possible Mars' satellites and found them successfully in 1877 .

The next $20^{t h}$ century and the beginning of $21^{s t}$ one turned out to be much more abundant in satellite discoveries due to both ground-based observations and space missions (Voyager-1, Voyager-2, Cassini-Huygens, Galileo). The Voyagers, which were launched in 1977 and flew by the Jupiter, Saturn, Uranus and Neptune, found 27 new satellites: 3 of Jupiter, 7 of Saturn, 11 of Uranus and 6 of Neptune. Six new moons of Saturn were discovered by Cassini-Huygens mission in the last five years. In 1993 the first asteroid satellite (Dactyl) orbiting the main-belt asteroid 243 Ida was discovered by the space mission Galileo. It was the beginning of abundant discoveries of asteroid satellites by photometric and radar observations and then of satellites of Kuiper-belt and transneptunian objects. The first minor planet with two satellites, 87 Sylvia, was identified by adaptive optics telescope direct imaging in 2005. Pluto's first satellite Charon was discovered in 1978. Other binary/multiple TNOs have been discovered since 2000. Since 15 May 2005 Pluto is known to be the only quadruple system.

\section{Modern world of the satellites and binaries}

Satellites of the planets. Six planets of the Solar System have satellites, total amount of which up-to-date is 168. Timeline of their discoveries is presented in Table 1. It is interesting to note, that the amount of satellites increased more than 2.5 times during the $20^{t h}$ century and was increased again 1.5 times during the first ten years of the $21^{\text {st }}$ century. Meanwhile, the space mission discoveries contribute about 20 percents. Table 2 contains the principal parameters (radius and mass) of seven biggest satellites in the Solar System.

Multiple systems among asteroids and transneptunian objects. By the end of February 2010 there were discovered 182 objects with companions: 172 binaries, 9 triple 
systems, and 1 quadruple system (Pluto). They count 193 companions total (see website "Asteroids with satellites" by Wm. R. Johnston www.johnstonsarchive.net/astro/ asteroidmoons.html).

These systems include the following:

- 37 near-Earth asteroids (2 NEAs with two satellites each);

- 9 Mars crossing asteroids (MCAs);

- 69 main-belt asteroids (5 MBAs with two satellites each);

- 4 Jupiter Trojan asteroids (JTAs);

- 63 transneptunian objects (2 of them 136108 Haumea and 471711999 TC36 with two and 134340 Pluto with three satellites - Charon, Nix and Hydra). Among these TNOs 3 objects (134340 Pluto, 136108 Haumea and 136199 Eris) now are recognized as dwarf planets under the current IAU nomenclature.

The near-Earth binary asteroids consist of 6 Atens, 21 Apollos, and 8 Amors, plus the triple Amor asteroid (153591) 2001 SN263 and the triple Apollo asteroid (136617) 1994 CC. A fraction of binary systems among the NEOs is estimated to be 15-17\% (Merline et al. 2002).

The first confirmed binary Trojan asteroid 617 Patroclus has very similar in size components. The $2^{\text {nd }}$ identified binary Trojan asteroid 624 Hektor has a primary which is a contact binary accompanied by a smaller secondary.

The first companion discovered among more than 1300 known TNOs and Centaurs was that of 1998 WW31, found in December 2000. Now the total number of multiple systems constitutes 60 binary TNOs, 2 triple TNO system, and the quadruple system of Pluto. Thus, as well as in case of asteroids, the multiple systems among TNOs seem to be relatively common.

One might note that only one satellite Dactyl was discovered due to the direct imaging by NASA space probe Galileo. The contribution of different methods to discoveries of companions is as following:

- photometric lightcurve method 41\% (48 MBAs, 14 NEAs, 9 MCAs, 2 JTAs and 1 $\mathrm{TNO})$;

- HST-imaging 30\% (3 MBAs and 51 TNOs);

- ground-based imaging 16.5\% (17 MBAs, 2 JTA and 11 TNOs);

- radar observations $12.5 \%$ (only $23 \mathrm{NEAs}$ ).

\section{Discussion and Conclusion}

In spite of the fact that all discoveries of satellites and binary systems among asteroids and transneptunian bodies are registered and cataloged, we cannot precisely count how many moons in total are discovered to date. The reason is that there is no established definition of what should be considered as a moon and it concerns first of all asteroid and transneptunian body populations. Indeed, there is no rule to discriminate between a double asteroid and asteroid with a satellite, both of which are binary systems. What binary system do we have to consider with certainty as a primary body and its satellite? What should be the upper limit of their mass ratio or at what minimum distance from primary the barycentre of binary system should be located? Some people assume that it should be located below the surface of the larger body, though this postulate is somewhat arbitrary and not generally accepted. Due to this uncertainty, for example, some authors consider the Pluto-Charon system to be a double (dwarf) planet.

The analysis of binary system parameters (which in general are not determined accurately enough) shows that the fraction of systems with nearly the same in sizes components $\left(\mathrm{R}_{\text {prm }} / \mathrm{R}_{\text {scd }} \leqslant 2\right)$ is $\sim 14 \%$ among the NEAs, $\sim 20 \%$ among the MBAs, $\sim 60 \%$ 
among the JTAs and $\sim 90 \%$ among the TNOs. It means that there is a strong observation selection effect in discovery of satellites: due to a weakness of asteroid's and especially of TNO's brightness it is much more complicated to detect a small companion near a bright primary than to detect two nearly equal in brightness components.

In any case, the population of discovered natural satellites, orbiting the large planets, numbered in 168 . Besides, roughly about $25 \%$ of all discovered binary asteroids and transneptunian bodies can be considered as having moons. Thus, one can conclude that about two hundred satellites are discovered in the Solar System to date.

They have a surprising variety of their orbits and physical properties. Nevertheless, it is impossible to overestimate the discovery of the first four Jupiter's moons Io, Europa, Ganymede and Callisto made by Galileo Galilei 400 years ago. Galileo's discovery of Jupiter moons had a major impact on cosmology of that time. In 1610 the traditional Aristotelian cosmology recognized only one center of motion, the center of the universe which was the place of the Earth. But according to the Copernican theory, the Earth went around the Sun while the Moon went around the Earth. Galileo discovered one more center of motion Jupiter, a new system of planetary bodies in miniature, and it was a strong argument in support of the new Copernican System.

Nowadays the discoveries of binary asteroids and TNOs objects give a good opportunity to determine their masses and bulk densities, and hence a type of their material and internal structure. However those estimates usually are still not accurate enough due to uncertainty of binary system parameters.

\section{Acknowledgements}

The authors are grateful to SOC and LOC of the IAU Symposium 269 for the IAU grants, which gave them the opportunity to participate in this unforgettable meeting in the Galileo's homeland.

\section{References}

Merline, W. J., Weidenschilling, S. J., Durda, D. D., Margot, J-L., Pravec, P., \& Storrs, A. D. 2002, Asteroids III. (W. F. Bottke et al., eds.), Univ. of Arizona Press, Tucson (USA), 289 